\title{
Novel compound heterozygous mutations T2C and 1149insT in the $K C N Q 1$ gene cause Jervell and Lange-Nielsen syndrome
}

\author{
RONG-RONG WANG ${ }^{1}$, NING LI ${ }^{1}$, YIN-HUI ZHANG ${ }^{1}$, LIN-LIN WANG $^{1}$, SI-YONG TENG ${ }^{2}$ and JIE-LIN PU ${ }^{1,2}$ \\ ${ }^{1}$ Pathology and Physiology Research Center and ${ }^{2}$ Center for Arrhythmia Diagnosis and Treatment, \\ Cardiovascular Institute and Fu Wai Hospital, Peking Union Medical College and Chinese \\ Academy of Medical Sciences, Xi-Cheng District, Beijing 100037, P.R. China
}

Received January 4, 2011; Accepted February 14, 2011

DOI: $10.3892 /$ ijmm.2011.642

\begin{abstract}
Mutations in the KCNQ1 gene account for more than $90 \%$ of the individuals with Jervell and Lange-Nielsen syndrome (JLNS). In this study, we identified and characterized two novel $K C N Q 1$ mutations that caused JLNS. A 6-year-old deaf girl suffering from recurrent syncope had a documented electrocardiogram with polymorphic ventricular fibrillation since the age of 4 years. The baseline electrocardiogram showed a significantly prolonged corrected QT interval (524 msec). Genetic analysis revealed that the proband carried two heterozygous mutations of T2C and 1149insT in the KCNQ1 gene on separate alleles. Patch-clamp analysis demonstrated that the T2C mutation resulted in significant reduction in the slowly activated delayed rectifier current $\left(I_{\mathrm{Ks}}\right)$. Furthermore, Western blot analysis and confocal imaging revealed that the $\mathrm{T} 2 \mathrm{C}$ mutation produced a truncated protein with trafficking defects. In contrast, the 1149insT mutation failed to generate any measurable current, consistent with no protein expression in both the cell membrane and cytoplasm. Moreover, co-expression of the T2C and 1149insT mutations significantly reduced the peak tail current density to $8.27 \%$ of the wild-type (WT) current value, while co-transfected WT channels with either T2C or 1149insT mutant channels produced comparable current and channel kinetics to that of WT channels. Our study demonstrates that the compound heterozygous mutations T2C and 1149insT cause the 'loss-offunction' of the $I_{\mathrm{Ks}}$ that may account for the clinical phenotype of the proband. Multiple mechanisms have been involved in the pathogenesis of 'loss-of-function' of $I_{\mathrm{Ks}}$.
\end{abstract}

Correspondence to: Dr Jie-Lin Pu, Pathology and Physiology Research Center, Cardiovascular Institute and Fu Wai Hospital, Peking Union Medical College and Chinese Academy of Medical Sciences, 167 Bei-Li-Shi Road, Xi-Cheng District, Beijing 100037, P.R. China

E-mail: jielinpu@yahoo.com

Key words: ventricular fibrillation, genetics, KCNQ1 gene, ion channels

\section{Introduction}

The Jervell and Lange-Nielsen syndrome (JLNS) is an autosomal recessively inherited disease clinically characterized by the presence of congenital bilateral sensorineural hearing loss, a markedly prolonged correct QT interval (QTc) on a documented electrocardiogram (ECG) and a high incidence of sudden death secondary to polymorphic ventricular arrhythmias (e.g. torsade de pointes, TdP) in childhood (1). Most of the arrhythmic events (95\%) are triggered by emotions or exercise (2). JLNS is caused by homozygous or compound heterozygous mutations in KCNQ1 and KCNE1 genes, which are responsible for encoding the slowly activated delayed rectifier current $\left(I_{\mathrm{Ks}}\right)(3-5)$. Mutations in the $K C N Q 1$ gene account for more than $90 \%$ of individuals with JLNS $(2,6)$.

$I_{\mathrm{Ks}}$ is the main repolarizing current at increased heart rates and is highly sensitive to catecholamine (7). Decrease in $I_{\mathrm{Ks}}$ may lead to prolongation of the action potential duration and thus, to the development of TdP (8). Functional studies of the mutant $K C N Q 1$ gene have indicated that multiple biophysical consequences led to the 'loss-of-function' of $I_{\mathrm{Ks}}$, including (i) a nonsense mutation resulting in the formation of non-functional channels, (ii) altered gating properties of the mutant channels, (iii) defective trafficking and dominant-negative effects, and (iv) disruption of channel assembly (9-12). In addition, recent reports have suggested that mutations involving the KCNQ1 gene could cause 'loss-of-function' of $I_{\mathrm{Ks}}$ by affecting the combination of $I_{\mathrm{Ks}}$ channels with other interacting protein $(13,14)$.

In the present study, we identified two novel compound heterozygous mutations, T2C and 1149insT, in the KCNQ1 gene in the female proband with JLNS. The proband inherited one of the mutations on separate alleles from each parent. The effects of these mutations on the function of $I_{\mathrm{Ks}}$ channels were studied. The 'loss-of-function' of the compound heterozygous mutant channels was suggested.

\section{Materials and methods}

Clinical examination. The proband and her first-degree relatives underwent clinical evaluation, including medical and family history reviews, physical examination, 12-lead ECG and echocardiography examinations (Fig. 1). The study 
complied with the Declaration of Helsinki and Institutional review board approvals. Written informed consents were obtained from all participants.

Genotyping. Genomic DNA was isolated from peripheral blood leukocytes using TIANamp Blood DNA isolation kit (Tiangen, Beijing, China). All the exons of the KCNQ1 gene were amplified by polymerase chain reaction (PCR) and were analyzed by direct sequencing. Whenever the mutation or SNP was found in the proband or her family members, it would be confirmed in 500 unrelated healthy Chinese individuals.

Mutagenesis and expression studies. The wild-type (WT)-KCNQ1 cDNA (PubMed Accession no. NM000218.2) was subcloned into a pcDNA3.1 expression vector. Two mutations, T2C-KCNQ1 and 1149insT-KCNQ1, were created by a site-directed mutagenesis strategy as previously described (15). Both mutations were verified by sequencing and subcloned back into pcDNA3.1 vectors. Chinese hamster ovary $(\mathrm{CHO})$ cells were transiently transfected with either $0.6 \mu \mathrm{g} \mathrm{WT}$ or an equal amount of mutant $K C N Q 1 \mathrm{cDNA}$ using the Effectene transfection reagent (Qiagen, Hilden, Germany). Green fluorescent protein $(G F P)$ plasmid $(0.2 \mu \mathrm{g})$ was co-transfected as an indicator. The transfected cells were cultured for $36 \mathrm{~h}$ before $I_{\mathrm{Ks}}$ was recorded. More than three independent transfection experiments were conducted for further patch-clamp and confocal experiments to confirm the reproducibility of the results.

Patch-clamp method. Membrane currents were measured using a whole-cell configuration of the patch-clamp technique with Axon Patch 700B amplifiers (Axon Instruments, Foster City, CA, USA). To stabilize the current, experiments were performed 5 min after entering the whole-cell configuration. All the measurements were obtained at room temperature $\left(22^{\circ} \mathrm{C}\right)$. The resistance of pipettes in the bath solution ranged from 2-4 M 2 . For whole-cell recording, the internal pipette solution contained (in mmol/l), K-ASP 120.0, $\mathrm{KCl}$ 20.0, $\mathrm{K}_{2} \mathrm{ATP} 4.0$, ethylene glycoltetetraacetic acid 10.0, $\mathrm{MgCl}_{2} 1.0, \mathrm{Na}_{2}$-phosphocreatin 2.0 and HEPES 5 (pH 7.4). The bath solution contained (in mmol/l), $\mathrm{NaCl} 136.0, \mathrm{KCl} 3.0$, $\mathrm{NaH}_{2} \mathrm{PO}_{4} \cdot 2 \mathrm{H}_{2} \mathrm{O} 0.33, \mathrm{MgCl}_{2} 1.0, \mathrm{CaCl}_{2} 1.8$, glucose 10.0 and HEPES 10.0 ( $\mathrm{pH}$ 7.4). No leak subtraction was applied during recording. $I_{\mathrm{Ks}}$ was elicited from a holding potential of $-40 \mathrm{mV}$ to a $5 \mathrm{sec}$ depolarized potential stepped from $-40 \mathrm{mV}$ to $+60 \mathrm{mV}$ with $10 \mathrm{mV}$ increments followed by a repolarization step of $2.5 \mathrm{sec}$ to $-40 \mathrm{mV}$. The current density was calculated by dividing the peak tail current by the cell capacitance. Voltage dependence of channel activation was obtained by fitting normalized curves with the Boltzmann function.

Immunocytochemistry. Cells were fixed for $15 \mathrm{~min}$ at room temperature with $4 \%$ paraformaldehyde $(\mathrm{pH} 7.4)$, and then were permeabilized with $0.2 \%$ Triton X-100 for $10 \mathrm{~min}$. After blocking the cells with $5 \%$ bovine serum albumin (BSA) for $25 \mathrm{~min}$ at room temperature, the cells were incubated with primary antibody against the $\mathrm{N}$ - or $\mathrm{C}$-terminus of the KCNQ1 channel protein (1:100 or 1:1000 dilution, respectively; Abcam, Cambridge, UK) overnight at $4^{\circ} \mathrm{C}$. The next day, the cells were rinsed with phosphate-buffered saline (PBS) followed by

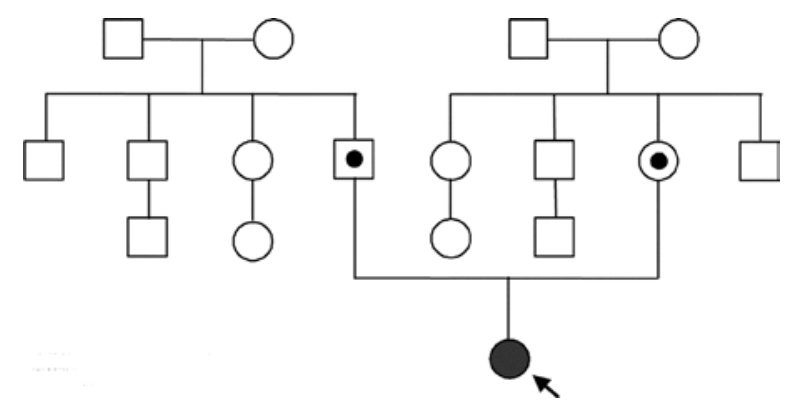

Figure 1. Pedigree of the family. Squares and circles represent male and female members, respectively. Solid circle (arrow) represents the proband carrying two compound heterozygous mutations of T2C and 1149insT in the $K C N Q 1$ gene on separate alleles. The black dots in the square or circle denote the parents carrying a single, but different heterozygous mutation.

incubation with a Cy3-conjugated secondary antibody (1:150 dilution, Jackson ImmunoResearch, West Grove, PA) for $1 \mathrm{~h}$ at room temperature in the dark. Finally, the coverslips were mounted with mounting medium (Zhongshan Golden Bridge Biotechnology Co., Ltd., Beijing, China). Confocal images were obtained using a confocal laser scanning microscope (Leica TCS SP2, Leica Microsystems, Wetzlar, Germany) and were analyzed with NIH image software, Image J.

Western blot analysis. The proteins from transfected CHO cells were separated using the M-PER mammalian protein extraction reagent (Pierce, Hilden, Germany) and Western blot analysis experiments were performed as previously described (16). Equal amounts of protein were run on $10 \%$ SDS-polyacrylamide gels and were transferred to a nitrocellulose membrane. The membrane was incubated with a primary antibody against the $\mathrm{N}$ - or C-terminus of the KCNQ1 channel protein (1:100 or 1:1000 dilution, respectively; Abcam) for $1 \mathrm{~h}$ at room temperature. Subsequently, the antibodies were visualized with a BCIP/NBT detection kit (Promega, Madison, USA).

Statistical analysis. The data are presented as the mean \pm SEM (standard error of the mean). Student's t-test analysis was performed for comparison of two means, while one-way ANOVA was performed for comparisons of multiple means using the statistical software, SPSS 13.0. A P-value $<0.05$ was considered statistically significant.

\section{Results}

Clinical manifestation and genotyping. The 6-year-old deaf girl, who suffered from recurrent syncope, had a documented ECG with polymorphic ventricular tachycardia or fibrillation since the age of 4 years. The syncope was always elicited by excitement or exercise and was spontaneously terminated. The baseline ECG showed a significantly prolonged QTc interval (524 msec) (Fig. 2A and D). No abnormalities were found in the biochemical or hematological blood tests or in the echocardiography examination. The audiometry revealed a bilateral symmetric sensorineural hypacusis. According to the clinical manifestations, JLNS was diagnosed and an electronic cochlear was implanted to restore hearing. After Betaloc administration, the proband remained symptom-free for a period of 2 years even with a significantly prolonged 

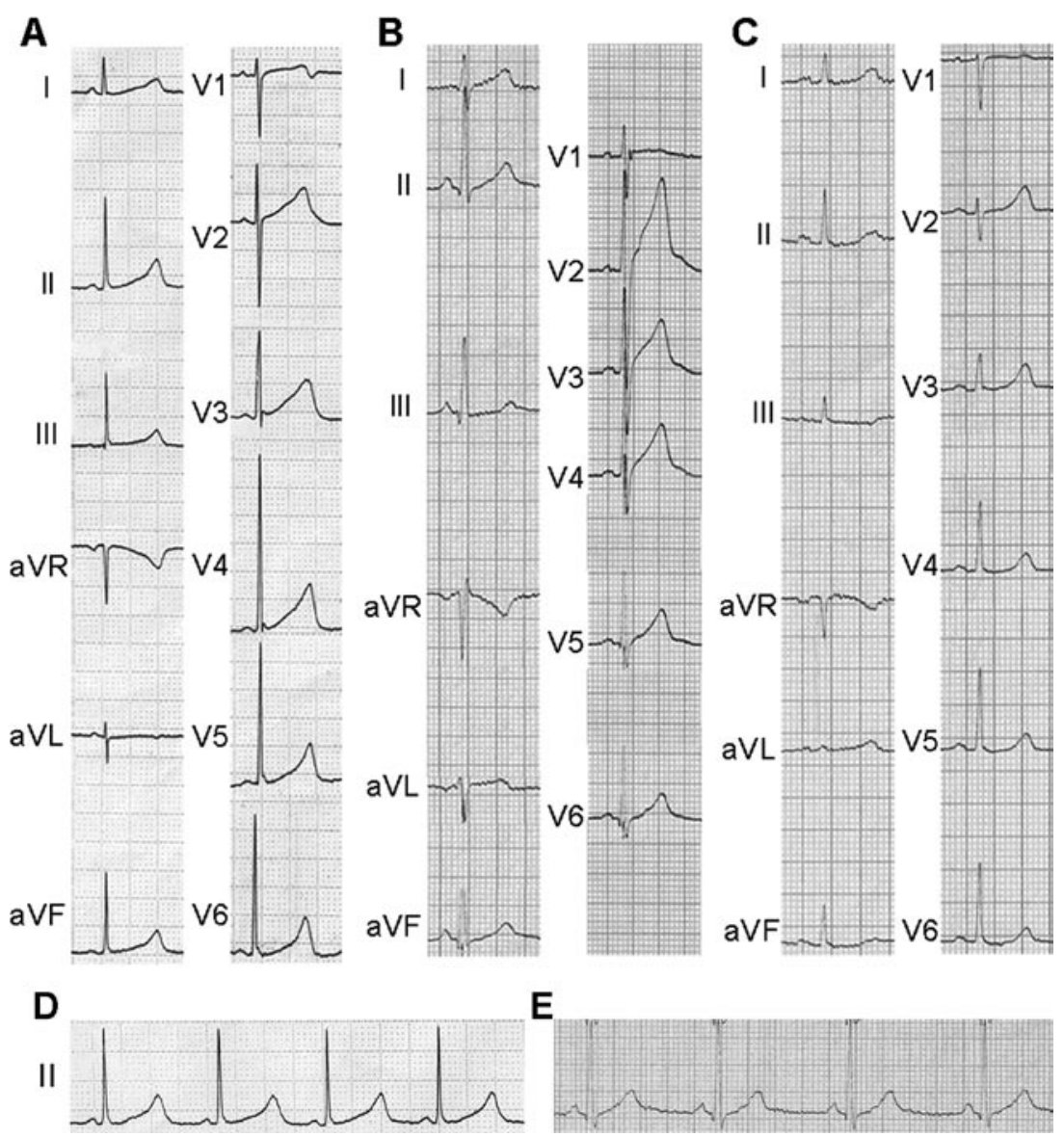

E

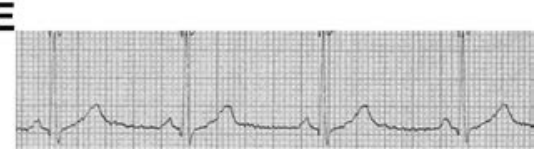

Figure 2. Twelve-lead ECG recordings of the proband and her family members. (A) The baseline ECG of the proband showed a markedly prolonged QTc interval ( $524 \mathrm{msec}$ ), whereas (B) the QTc intervals were $460 \mathrm{msec}$ (borderline) on her father's ECG and (C) $420 \mathrm{msec}$ (normal) on her mother's ECG. Plots displaying the ECG leads II of (D) the proband and (E) her father.

QTc interval of $624 \mathrm{msec}$. Her parents were free of hearing impairment or ventricular arrhythmias even during exercise. The QTc intervals were $460 \mathrm{msec}$ (borderline) on her father's ECG (Fig. 2B and E) and 420 msec (normal) on her mother's ECG (Fig. 2C). There was no family history of sudden death. Detail data from other family members on both sides were unavailable due to lack of the written informed consents.

Genetic analysis revealed that the proband carried two mutations in exons 1 and 9 of the KCNQ1 gene on separate alleles. The paternal allele had a substitution of a $\mathrm{C}$ for a $\mathrm{T}$ at position 2 (T2C) in exon 1 of the $K C N Q 1$ gene. The nucleotide change caused a substitution of threonine (Thr) for the initiation codon at position 1 of the KCNQ1 protein (Fig. 3A and C). In contrast, exon 9 of the $K C N Q 1$ gene on the maternal allele displayed an insertion of $\mathrm{T}$ at position 1149 (1149insT), which caused a frameshift at codon 384 resulting in introduction of a premature termination codon (PTC) at codon 462 that was predicted to form a non-functional truncated protein (A384fs/79) (Fig. 3B and C) or in the absence of protein formation because of the existence of a nonsense-mediated mRNA decay (NMD) mechanism (17). Neither the T2C nor the 1149insT mutation was detected in 500 healthy unrelated subjects.

Electrophysiological characteristics. $I_{\mathrm{Ks}}$ was recorded from the CHO cells transiently transfected with WT or mutant channels using whole-cell configuration of the patch-clamp
A

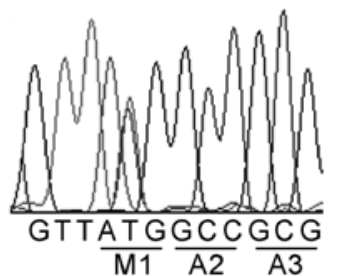

$\mathrm{MU}$

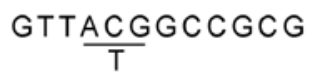

B

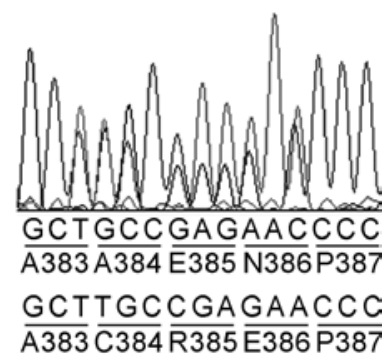

C

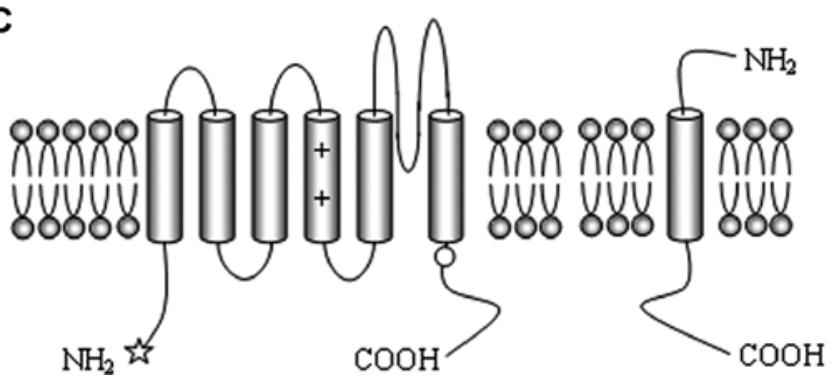

Figure 3. (A) DNA sequence analysis of the proband and her father identified a substitution of a $\mathrm{C}$ for a $\mathrm{T}$ at position 2 of the coding region of the $K C N Q 1$ gene leading to an initiation codon with a threonine substitution at an $\alpha$ subunit of the $I_{\mathrm{Ks}}$ channels. (B) DNA sequence analysis of the proband and her mother showed an insertion of $\mathrm{T}$ at position 1149 causing a frameshift at codon 384 that resulted in a premature stop codon at position 462 . (C) The $\alpha$ subunit of the $I_{\mathrm{Ks}}$ channels is a transmembrane protein with six transmembrane segments. ( $(\vec{\aleph})$ Indicates the location of the initiation codon, and (o) denotes the 384 amino acid residues. 
A
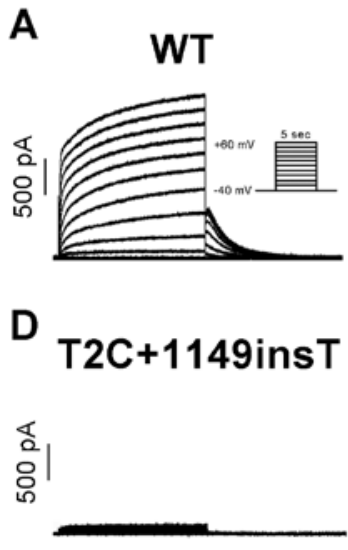

G

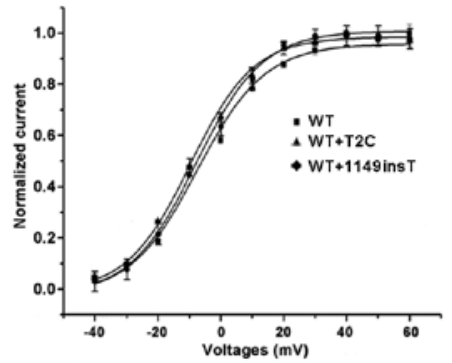

B

T2C

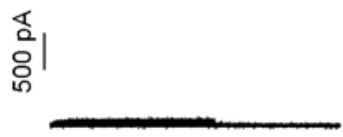

E

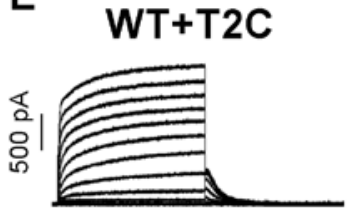

C

1149ins T

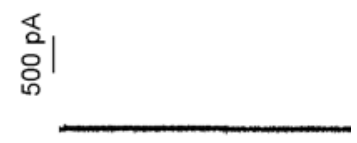

$\mathbf{F}$

WT+1149ins T

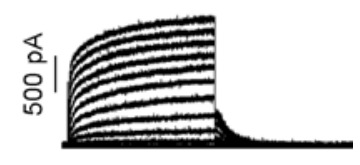

H

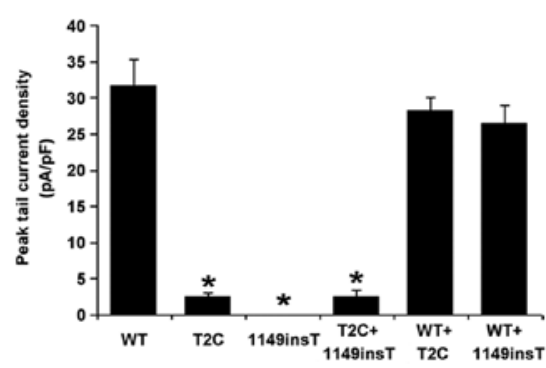

Figure 4. Current traces recorded from the CHO cells transfected transiently with (A) WT, (B) T2C, (C) 1149insT or co-transfected with (D) T2C+1149insT, or (E) WT+T2C or (F) with 1149insT mutant. $I_{\mathrm{Ks}}$ was elicited from a holding potential of $-40 \mathrm{mV}$ to a $5 \mathrm{sec}$ depolarized potential stepped from $-40 \mathrm{mV}$ to $+60 \mathrm{mV}$ with $10 \mathrm{mV}$ increments followed by a repolarization step of $2.5 \mathrm{sec}$ to $-40 \mathrm{mV}$. (G) Voltage dependence of the activation curve of WT (घ), WT+T2C (₫) and WT+1149insT (•) channels. The $\mathrm{V}_{1 / 2}$ (the voltage at which $50 \%$ of channels are activated) was $-7.39 \pm 0.93,-8.03 \pm 0.68$ and $-9.05 \pm 1.53 \mathrm{mV}$, while $\mathrm{k}$ was $10.80 \pm 1.67,10.94 \pm 1.72$ and $11.04 \pm 2.08$, respectively (all $\mathrm{P}>0.05$ ). (H) Peak tail current densities under the background of WT, mutant, or heterozygous expression. The peak tail current density of T2C or 1149insT mutant channels was dramatically decreased or completely diminished compared to that of WT channels, while the tail current densities of WT, WT+T2C and WT+1149insT channels were comparable $(\mathrm{P}>0.05)$.

technique. The peak tail current density of the T2C mutant channels was dramatically reduced compared to that of the WT channels $(31.67 \pm 3.64 \mathrm{pA} / \mathrm{pF}$ in WT, $\mathrm{n}=7$ vs. $2.50 \pm 0.58 \mathrm{pA} / \mathrm{pF}$ in T2C mutant, $\mathrm{n}=7, \mathrm{P}<0.05$, Fig. $4 \mathrm{~A}$ and $\mathrm{B})$. In contrast, the 1149insT mutant channels failed to generate any measurable $I_{\mathrm{Ks}}$, indicating that the 1149insT mutation completely abolished the $I_{\mathrm{Ks}}$ (Fig. 4C). Furthermore, co-transfection of T2C with 1149insT mutant channels (as the genotype of the proband) significantly reduced the peak tail current density to $8.27 \%$ of that of WT channels $(2.62 \pm 0.79 \mathrm{pA} / \mathrm{pF}, \mathrm{n}=6, \mathrm{P}<0.05$, Fig. 4D). However, co-expression of WT-KCNQ1 with either T2C or 1149insT mutant (as the genotype of her father or mother) produced the channels with comparable current densities $(28.26 \pm 1.73 \mathrm{pA} / \mathrm{pF}, \mathrm{n}=5$ vs. $26.43 \pm 2.49 \mathrm{pA} / \mathrm{pF}, \mathrm{n}=5, \mathrm{P}>0.05)$ and kinetics to those of the WT channels, suggesting that T2C or 1149insT mutation had no dominant negative effect on WT channel function (Fig. 4E-G). The peak tail current densities were plotted in Fig. 4H for all combinations of WT-KCNQ1, T2C-KCNQ1, and 1149insT-KCNQ1 constructs.

Confocal imaging. To investigate the cellular localization of the WT and mutant channels, immunocytochemistry experiments were performed. The non-transfected cells showed no fluorescence. As expected, in the cells expressing the WT channels, strong red fluorescence was visualized predominantly on the cell membrane. However, the peripheral localization was disrupted in the cells transfected with T2C mutant channels. Instead, the T2C mutant channels displayed a restricted perinuclear distribution detected by a primary antibody against the C-terminus of the KCNQ1 channels. In contrast, the 1149insT mutant channels showed no expression in both the cell membrane and cytoplasm assessed by a primary antibody against the $\mathrm{N}$ - or C-terminus of the KCNQ1 channels. In addition, cells co-expressing the T2C and the 1149insT mutant channels showed the same distribution pattern as that of the T2C channels (Fig. 5B and C).

Western blot analysis. The expression of WT or T2C mutant channel protein was also analyzed by Western blot analysis using a KCNQ1 C-terminal antibody. One band at around $72 \mathrm{kDa}$ was observed in the WT-KCNQ1 lane, representing the KCNQ1 channel protein, while the other band at around $55 \mathrm{kDa}$ was detected in the T2C-KCNQ1 lane, signifying that the mutation in the initiation codon led to an alternate activation of the initiation site in the mRNA of $K C N Q 1$ gene. However, no band was observed in the 1149insT-KCNQ1 lane detected by a KCNQ1 N-terminal antibody, consistent with minimal expression levels of the 1149insT mutant channels both on the cell membrane and in the cytoplasm (Fig. 5A).

\section{Discussion}

In the present study, we identified a novel T2C mutation in the initiation codon of the $K C N Q 1$ gene that dramatically 

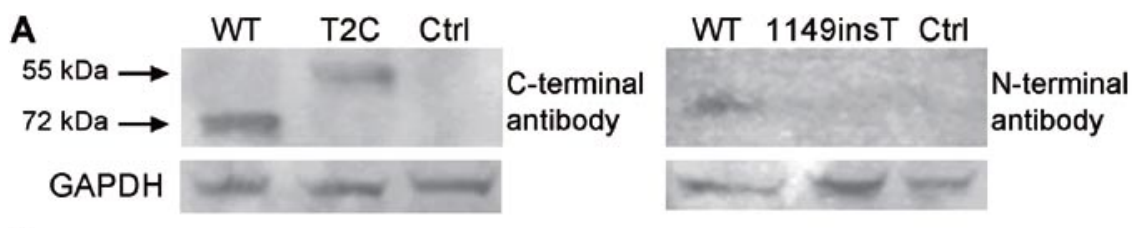

B KCNQ1 N-terminal antibody

WT
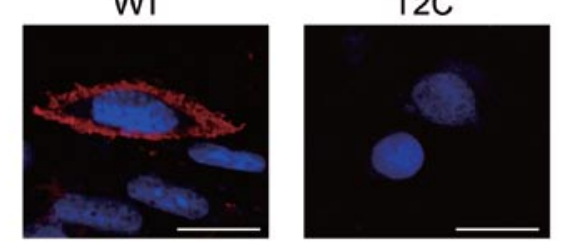

C

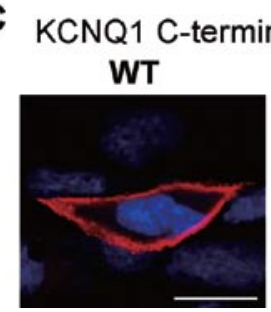

1149insT

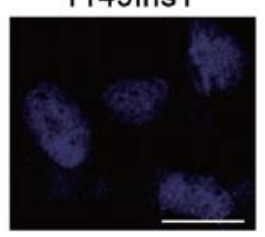

1149ins T

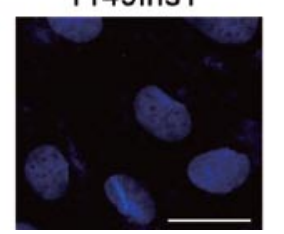

Control

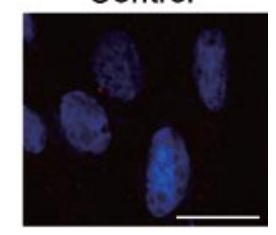

T2C+1149ins T

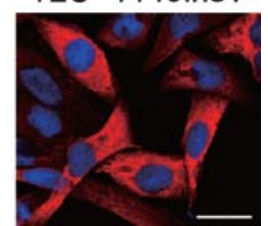

Figure 5. Western blot analysis and confocal imaging of WT and mutant KCNQ1 channels. (A) T2C-KCNQ1 cDNA generated a shorter protein product of $55 \mathrm{kDa}$ compare to WT-KCNQ1 protein (72 kDa) using a KCNQ1 C-terminal antibody, while no protein band was detected in the 1149insT-KCNQ1 lane using a KCNQ1 N-terminal antibody. (B) The cellular localization of the WT or mutant channels was detected in cells transfected with the WT, T2C or 1149insT construct using an N-terminal antibody. Strong red fluorescence was visualized on the membrane of the cells expressing WT channels, while the cells transfected with the T2C or 1149insT constructs showed only background fluorescence. Blue fluorescence indicates the nuclei. (C) The fluorescence in cells was detected by a C-terminal antibody. The peripheral localization was exhibited in cells with WT channels. However, the cells expressing the T2C mutant channels or co-expressing the T2C and 1149insT mutant channels displayed a restricted perinuclear distribution. In contrast, the cells expressing the 1149insT mutant channels did not show fluorescence in the cell membrane or the cytoplasm. Bar, $20 \mu \mathrm{m}$.

reduced the $I_{\mathrm{Ks}}$. Western blot analysis studies showed that the T2C-KCNQ1 cDNA generated a shorter protein product of $55 \mathrm{kDa}$ compared to the WT protein $(72 \mathrm{kDa})$. Based on the molecular weight of the truncated protein and the sequence of the $K C N Q 1$ gene, we hypothesized that because of the mutation in the original translation initiation site, the protein synthesis might start from the second initiation site at position 583 in exon 2 of $K C N Q 1$ gene, generating a truncated protein missing the initial 158 residues compared to the full length of KCNQ1 protein. The mechanism was first described in the inherited arrhythmia syndromes, although it has been observed in other diseases (18-20). Ozisik et al found that an alternate translation initiation site circumvented an aminoterminal $D A X 1$ nonsense mutation leading to a mild form of $\mathrm{X}$-linked adrenal hypoplasia congenital (18). The other interesting finding was that the level of the truncated protein was less than that of the WT protein as determined by Western blot analysis experiments. The possible reasons may be that (i) although the second ATG codon in exon 2 of the $K C N Q 1$ gene could act as an initiation codon, the translation process from the internal methionine may be less effective, and (ii) that the truncated protein was unstable. Other unknown mechanisms responsible for the reduced expression of the truncated protein can not be excluded.

Furthermore, the immunocytochemistry experiments revealed that the T2C mutant channels were retained in endoplasmic reticulum, and thus displayed a restricted perinuclear distribution, indicating that the $\mathrm{T} 2 \mathrm{C}$ mutation predisposed the $I_{\mathrm{Ks}}$ channels to a 'loss-of-function' due to a trafficking defect.
However, the mechanism of the retention of the T2C mutant channels is not fully understood. Sequence analysis showed that the $\mathrm{N}$-terminus of the $K C N Q 1$ gene harbors arginine (12-RKR-14, 24-RR-25, 97-RR-98), leucine (38-LEL-40), and tyrosine (-51-YAPI-54, 111-YNFL-114) based motifs together with a proline rich domain (51-P//P-89) which have been well-known as transport signals involved in the trafficking of several membrane proteins (21-23). However, Dahimène et al reported that the $\mathrm{N}$-terminal juxtamembranous domain preceding the first transmembrane domain of the protein rather than the initial 106-amino acids of the KCNQ1 protein was critical for channel surface expression (24). Consistently, the $K C N Q 1$ isoform 2, a truncated splice variant missing the 128 initial residues of KCNQ1 protein, displayed a perinuclear distribution because of the trafficking defect (24). Thus, in the present study, the $\mathrm{T} 2 \mathrm{C}$ mutation in the initiation codon might cause an alternate translation initiation site downstream of the mutation, yielding a truncated protein missing the 158 initial residues of the full length of the KCNQ1 channel, and finally leading to 'loss-of-function' of the mutant channels due to a trafficking defect.

In addition, we found another new frameshift mutation, 1149 insT, in the $K C N Q 1$ gene that caused a frameshift at codon 384 leading to introduction of a PTC at position 462 of the mutant channels. Functional consequences of the mutation revealed that it failed to generate measurable $I_{\mathrm{Ks}}$, consistent with complete inhibition of the expression of the mutant channels in both the cytoplasm and cell membrane detected by immunocytochemistry experiments. Several studies have 
showed that nonsense or frameshift mutations bearing PTCs can destabilize mRNA transcripts via a mechanism known as NMD in several human diseases $(17,25,26)$. According to the proposed rule, when the translation termination codons located more than 50-55 nucleotides upstream of the 3'-most exon-exon junction, NMD mechanism could occur $(27,28)$. In our study, the introduced PTC harbored at position 1384 of the mRNA transcripts was far upstream from the 3'-most exon-exon junction, therefore, we speculated that the 1149insT mutation might lead to 'loss-of-function' of the mutant channels via the NMD mechanism.

Furthermore, we expressed T2C and 1149insT cDNA constructs to simulate the genotypes of the proband and her family. Co-transfected T2C and 1149insT mutation (as the genotype of the proband) significantly reduced the peak tail current density to $8.27 \%$ of the WT values, consistent with the severe clinical phenotype of the proband. However, co-expressing the same amount of WT and T2C cDNA (as the genotype of her father), or WT and 1149insT cDNA (as the genotype of her mother) produced $I_{\mathrm{Ks}}$ densities similar to those of the WT cDNA, indicating that the T2C or 1149insT mutant protein did not have a dominant negative effect on the WT channels. The results might explain the mild or normal clinical phenotype of the heterozygous family members. In conclusion, our study demonstrated that the compound heterozygous mutations T2C and 1149insT led to 'loss-of function' of the $I_{\mathrm{Ks}}$ channels that may account for the clinic phenotype of JLNS of the proband. Therefore, multiple mechanisms may be involved in the pathogenesis of 'loss-offunction' of $I_{\mathrm{Ks}}$.

\section{Acknowledgements}

We thank Dr Xinjie Bao (Peking Union Medical College, Beijing, China) for assistance with the confocal laser-scanning microscope. This work was supported by the '973' National Basic Research Program of China (2007CB512000 and 2007CB512008 to Dr Jie-Lin Pu.

\section{References}

1. Jervell A and Lange-Nielsen F: Congenital deaf-mutism, functional heart disease with prolongation of the Q-T interval, and sudden death. Am Heart J 54: 59-68, 1957.

2. Schwartz PJ, Spazzolini C, Crotti L, et al: The Jervell and LangeNielsen syndrome: natural history, molecular basis, and clinical outcome. Circulation 113: 783-790, 2006.

3. Moss AJ, Shimizu W, Wilde AA, et al: Clinical aspects of type-1 long-QT syndrome by location, coding type, and biophysical function of mutations involving the KCNQ1 gene. Circulation 115: 2481-2489, 2007.

4. Ning L, Moss AJ, Zareba W, Robinson J, Rosero S, Ryan D and Qi M: Novel compound heterozygous mutations in the KCNQ1 gene associated with autosomal recessive long QT syndrome (Jervell and Lange-Nielsen syndrome). Ann Noninvasive Electrocardiol 8: 246-250, 2003.

5. Tyson J, Tranebjaerg L, Bellman S, et al: IsK and KvLQT1: mutation in either of the two subunits of the slow component of the delayed rectifier potassium channel can cause Jervell and Lange-Nielsen syndrome. Hum Mol Genet 6: 2179-2185, 1997.

6. Berge KE, Haugaa KH, Früh A, et al: Molecular genetic analysis of long QT syndrome in Norway indicating a high prevalence of heterozygous mutation carriers. Scand J Clin Lab Invest 68: 362-368, 2008

7. Sanguinetti MC: Long QT syndrome: ionic basis and arrhythmia mechanism in long QT syndrome type 1. J Cardiovasc Electrophysiol 11: 710-712, 2000.
8. Aizawa Y, Ueda K, Scornik F, et al: A novel mutation in KCNQ1 associated with a potent dominant negative effect as the basis for the LQT1 form of the long QT syndrome. J Cardiovasc Electrophysiol 18: 972-977, 2007.

9. Henrion U, Strutz-Seebohm N, Duszenko M, Lang F and Seebohm G: Long QT syndrome-associated mutations in the voltage sensor of $\mathrm{I}(\mathrm{Ks})$ channels. Cell Physiol Biochem 24: $11-16,2009$

10. Wilson AJ, Quinn KV, Graves FM, Bitner-Glindzicz M and Tinker A: Abnormal KCNQ1 trafficking influences disease pathogenesis in hereditary long QT syndromes (LQT1). Cardiovasc Res 67: 476-486, 2005.

11. Labro AJ, Boulet IR, Timmermans JP, Ottschytsch N and Snyders DJ: The rate-dependent biophysical properties of the LQT1 H258R mutant are counteracted by a dominant negative effect on channel trafficking. J Mol Cell Cardiol 48: 1096-1104, 2010.

12. Schmitt N, Schwarz M, Peretz A, Abitbol I, Attali B and Pongs O: A recessive C-terminal Jervell and Lange-Nielsen mutation of the KCNQ1 channel impairs subunit assembly. EMBO J 19: 332-340, 2000.

13. Park KH, Piron J, Dahimene S, Mérot J, Baró I, Escande D and Loussouarn G: Impaired KCNQ1-KCNE1 and phosphatidylinositol-4,5-bisphosphate interaction underlies the long QT syndrome. Circ Res 96: 730-739, 2005.

14. Marx SO, Kurokawa J, Reiken S, Motoike H, D'Armiento J, Marks AR and Kass RS: Requirement of a macromolecular signaling complex for beta adrenergic receptor modulation of the KCNQ1-KCNE1 potassium channel. Science 295: 496-499, 2002.

15. Teng S, Gao L, Paajanen V, Pu J and Fan Z: Readthrough of nonsense mutation W822X in the SCN5A gene can effectively restore expression of cardiac $\mathrm{Na}^{+}$channels. Cardiovasc Res 83: 473-480, 2009.

16. Yao Y, Teng S,Li N, Zhang Y,Boyden PA and Pu J: Aminoglycoside antibiotics restore functional expression of truncated HERG channels produced by nonsense mutations. Heart Rhythm 6: 553-560, 2009.

17. Gong Q, Zhang L, Vincent GM, Horne BD and Zhou Z: Nonsense mutations in hERG cause a decrease in mutant mRNA transcripts by nonsense-mediated mRNA decay in human long-QT syndrome. Circulation 116: 17-24, 2007.

18. Ozisik G, Mantovani G, Achermann JC, et al: An alternate translation initiation site circumvents an amino-terminal DAX1 nonsense mutation leading to a mild form of X-linked adrenal hypoplasia congenital. J Clin Endocrinol Metab 88: 417-423, 2003.

19. Du W, Kumaki S, Uchiyama T, et al: A second-site mutation in the initiation codon of WAS (WASP) results in expansion of subsets of lymphocytes in an Wiskott-Aldrich syndrome patient. Hum Mutat 27: 370-375, 2006.

20. Gurvich OL, Maiti B, Weiss RB, Aggarwal G, Howard MT and Flanigan KM: DMD exon 1 truncating point mutations: amelioration of phenotype by alternative translation initiation in exon 6. Hum Mutat 30: 633-640, 2009.

21. Zerangue N, Schwappach B, Jan YN and Jan LY: A new ER trafficking signal regulates the subunit stoichiometry of plasma membrane K (ATP) channels. Neuron 22: 537-548, 1999.

22. Madrid R, Le Maout S, Barrault MB, Janvier K, Benichou S and Mérot J: Polarized trafficking and surface expression of the AQP4 water channel are coordinated by serial and regulated interactions with different clathrin-adaptor complexes. EMBO J 20: 7008-7021, 2001.

23. Ma D, Zerangue N, Lin YF, Collins A, Yu M, Jan YN and Jan LY: Role of ER export signals in controlling surface potassium channel numbers. Science 291: 316-319, 2001.

24. Dahimène $\mathrm{S}$, Alcoléa $\mathrm{S}$, Naud $\mathrm{P}$, et al: The $\mathrm{N}$-terminal juxtamembranous domain of KCNQ1 is critical for channel surface expression: implications in the Romano-Ward LQT1 syndrome. Circ Res 99: 1076-1083, 2006.

25. Holbrook JA, Neu-Yilik G, Hentze MW and Kulozik AE: Nonsense-mediated decay approaches the clinic. Nat Genet 36: 801-808, 2004

26. Bhuvanagiri M, Schlitter AM, Hentze MW and Kulozik AE: NMD: RNA biology meets human genetic medicine. Biochem J 430: 365-377, 2010.

27. Nagy E and Maquat LE: A rule for termination-codon position within intron-containing genes: when nonsense affects RNA abundance. Trends Biochem Sci 23: 198-199, 1998.

28. Maquat LE: Nonsense-mediated mRNA decay: splicing, translation and mRNP dynamics. Nat Rev Mol Cell Biol 5: 89-99, 2004. 